\title{
Case Report: Management of Malignancy-Exacerbated Pemphigus Vulgaris During COVID-19 Pandemic
}

\author{
Alberto Corrà ${ }^{1 *}$, Francesca Cammelli ${ }^{2}$, Lavinia Quintarelli ${ }^{1,3}$, Giuseppe Barbato ${ }^{2}$, \\ Ornella Le Rose ${ }^{4}$, Adele Salemme ${ }^{5}$, Giovanni Di Zenzo ${ }^{5}$, Francesco Coratti ${ }^{2}$, \\ Alice Verdelli ${ }^{1,3}$, Cristina Aimo ${ }^{1}$, Elena Biancamaria Mariotti ${ }^{1}$, Beatrice Bianchi ${ }^{1}$, \\ Fabio Cianchi ${ }^{2}$ and Marzia Caproni ${ }^{1,3}$
}

${ }^{1}$ Section of Dermatology, Department of Health Sciences, University of Florence, Florence, Italy, ${ }^{2}$ Division of Gastrointestinal Surgery, Careggi University Hospital, Azienda Ospedaliero-Universitaria Careggi, Florence, Italy, ${ }^{3}$ Rare Diseases Unit, Section of Dermatology, Department of Health Sciences, Azienda USL Toscana Centro, University of Florence, European Reference Network-Skin Member, Florence, Italy, ${ }^{4}$ Section of Radiology, Unità Sanitaria Locale Toscana Centro, "Piero Palagi" Hospital, Florence, Italy, ${ }^{5}$ Molecular and Cell Biology Laboratory, Istituto Dermopatico dell'Immacolata - Istituto di Ricovero e Cura a Carattere Scientifico, Rome, Italy

OPEN ACCESS

Edited by:

Ralf J. Ludwig,

University of Lübeck, Germany

Reviewed by:

Sergei Grando,

University of California, Irvine,

United States

Aikaterini Patsatsi,

Aristotle University of

Thessaloniki, Greece

*Correspondence:

Alberto Corrà

alberto.corra@unifi.it

Specialty section:

This article was submitted to Dermatology,

a section of the journal

Frontiers in Medicine

Received: 11 May 2021

Accepted: 05 July 2021

Published: 11 August 2021

Citation:

Corrà A, Cammelli F, Quintarelli L, Barbato G, Le Rose O, Salemme A, Di Zenzo G, Coratti F, Verdelli A, Aimo C, Mariotti EB, Bianchi B, Cianchi F and

Caproni M (2021) Case Report:

Management of

Malignancy-Exacerbated Pemphigus Vulgaris During COVID-19 Pandemic.

Front. Med. 8:708284.

doi: 10.3389/fmed.2021.708284
Pemphigus vulgaris is an intraepidermal autoimmune mucocutaneous blistering disease whose etiopathogenesis includes various trigger factors, i.e., drugs and malignancies. We present a case of malignancy-exacerbated pemphigus vulgaris which required a careful diagnostic process in order to rule out paraneoplastic pemphigus, along with the challenges posed by the need of treating both cutaneous and oncologic diseases. Possible post-operative complications post-poned the start of first-line immunosuppressive treatment of pemphigus. Moreover, the infective risks had to be minimized during the peak of the COVID-19 pandemic in Italy. Intravenous immunoglobulins were chosen as "bridge" therapy before the tumor surgical excision, followed by rituximab in post-operative phase.

Keywords: esophageal cancer, pemphigus vulgaris, rituximab, intravenous immunoglobulin, immunoblot, paraneoplastic pemphigus, COVID-19, Ivor Lewis esophagectomy

\section{INTRODUCTION}

Pemphigus is a potentially fatal autoimmune blistering disease which affects both mucous membranes and the skin. It is characterized by IgG autoantibodies targeting desmoglein 3 and/or desmoglein 1, which are molecules involved in the adhesion between keratinocytes. Antibody/antigen binding results in the loss of the adhesion functions of desmogleins and intraepidermal blistering. Pemphigus has been shown to occur following various triggers, including drugs and malignancies. Esophageal cancer is one of the leading malignant diseases and the sixth most common cause of cancer-related death worldwide (1). We wish to present a case of a patient with a mild pemphigus vulgaris (PV) dramatically exacerbated following a diagnosis of a solid tumor of the gastrointestinal tract.

\section{CASE REPORT}

An 80-year-old man was referred to our clinic due to a 6-year history of PV. He previously received treatments with systemic corticosteroids and immunosuppressants, including azathioprine and mycophenolate mofetil, both pre-maturely interrupted after 6 and 4 months, respectively, due to 

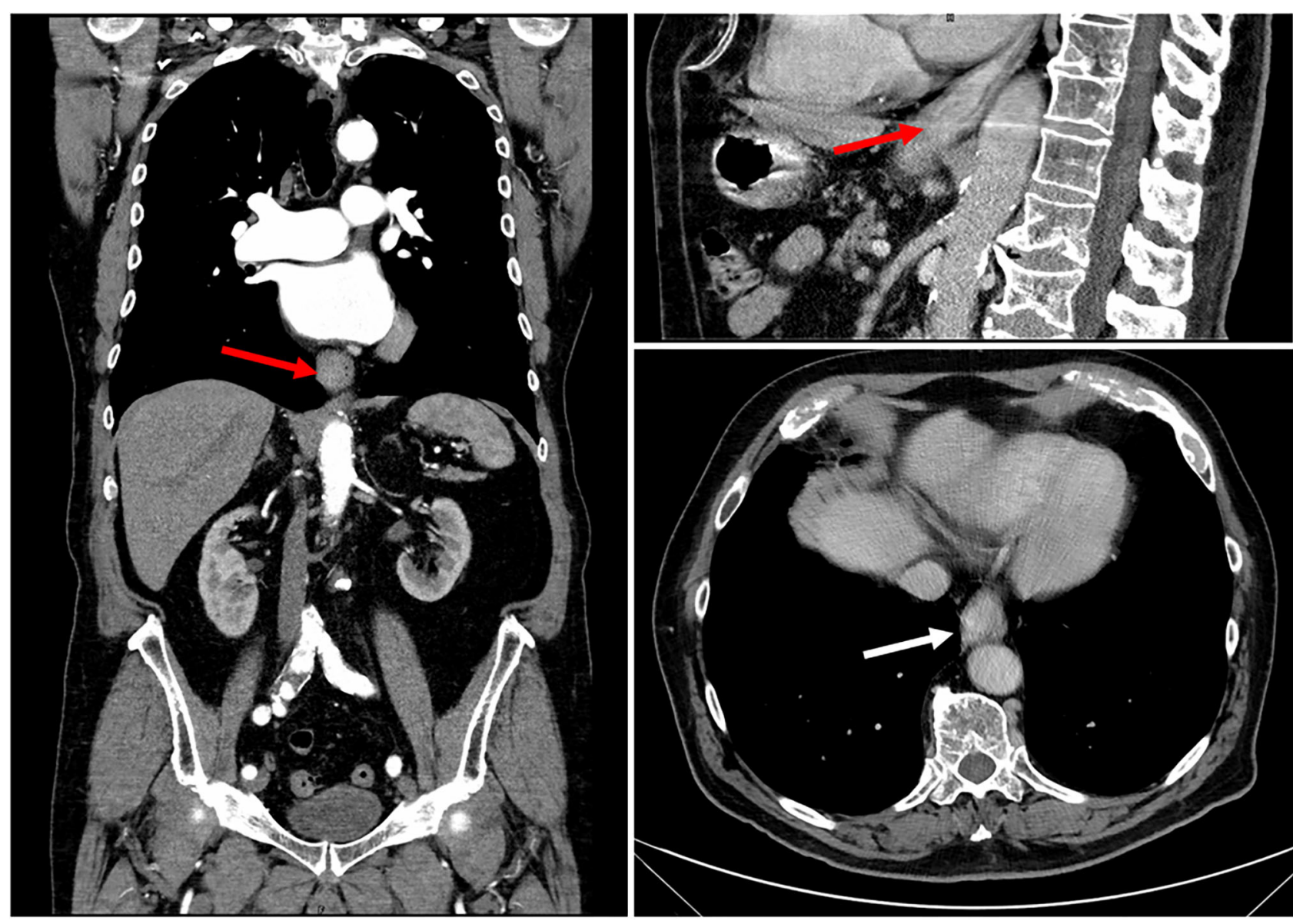

FIGURE 1 | CT scan of the esophageal mass showing asymmetrical neoplastic wall thickening, extending from the lower third of esophagus to the cardia (white arrow in axial view and red arrows in sagittal and coronal views). The mass caused esophageal stenosis without dilatation of the upstream lumen.

inefficacy. A cycle of rituximab, followed by two maintenance administrations 6 and 12 months later, had revealed effective in establishing a prolonged remission off-therapy for about 2 years. He had an episode of thrombophlebitis of the lower limbs 2 years earlier, for which he was on treatment with rivaroxaban. At the time of the first dermatological visit, the physical examination showed erosions mainly affecting the trunk and scalp. The Pemphigus Disease Activity Index (PDAI) was 7. Because of the low disease activity, a treatment with topical steroids was attempted.

After 4 weeks, the patient returned because of a significant worsening of his cutaneous lesions, which were enlarging and increasing in number, located in the head and neck, trunk, and limbs, with no mucosal sites involved. Pemphigus exacerbation was associated with the onset of other symptoms, including a progressive dysphagia, weight loss, and melena. A periodic re-assessment of serum autoantibody concentration also indicated a significant increase in both anti-Dsg1 and anti-Dsg3 antibody titers. An endoscopic examination of the distal esophagus indeed revealed a stenosing esophageal mass; a PET/CT scan confirmed the presence of a lesion $3 \mathrm{~cm}$ in diameter, highlighting metabolic activity areas corresponding only to tumor location, without nodal or distant metastasis (Figure 1). Previously, the chest RX and abdominal ultrasound conducted as screening for immunosuppressive treatments 2 years before were unremarkable, as well as the routine blood tests performed at that time.

The Gastrointestinal Surgery Unit opted for endoscopic resection of the tumor. In order to rule out paraneoplastic pemphigus (PNP), blood samples were collected for indirect immunofluorescence (IIF) on rat bladder and normal human skin and immunoblot for anti-plakin and antidesmocollin autoantibodies, which resulted negative (Figure 2; Supplementary Figures 1-3).

Before the surgical intervention, delayed due to the COVID19 outbreak, PV was managed with two cycles of intravenous immunoglobulins (IVIg, $2 \mathrm{~g} / \mathrm{kg}$ per cycle) 4 weeks apart. The prolonged treatment with systemic steroids before major surgical interventions is associated with a significant risk of surgical complications; thereby, systemic steroids were not administered. After two cycles of IVIg treatment, the PDAI score remained stable. 


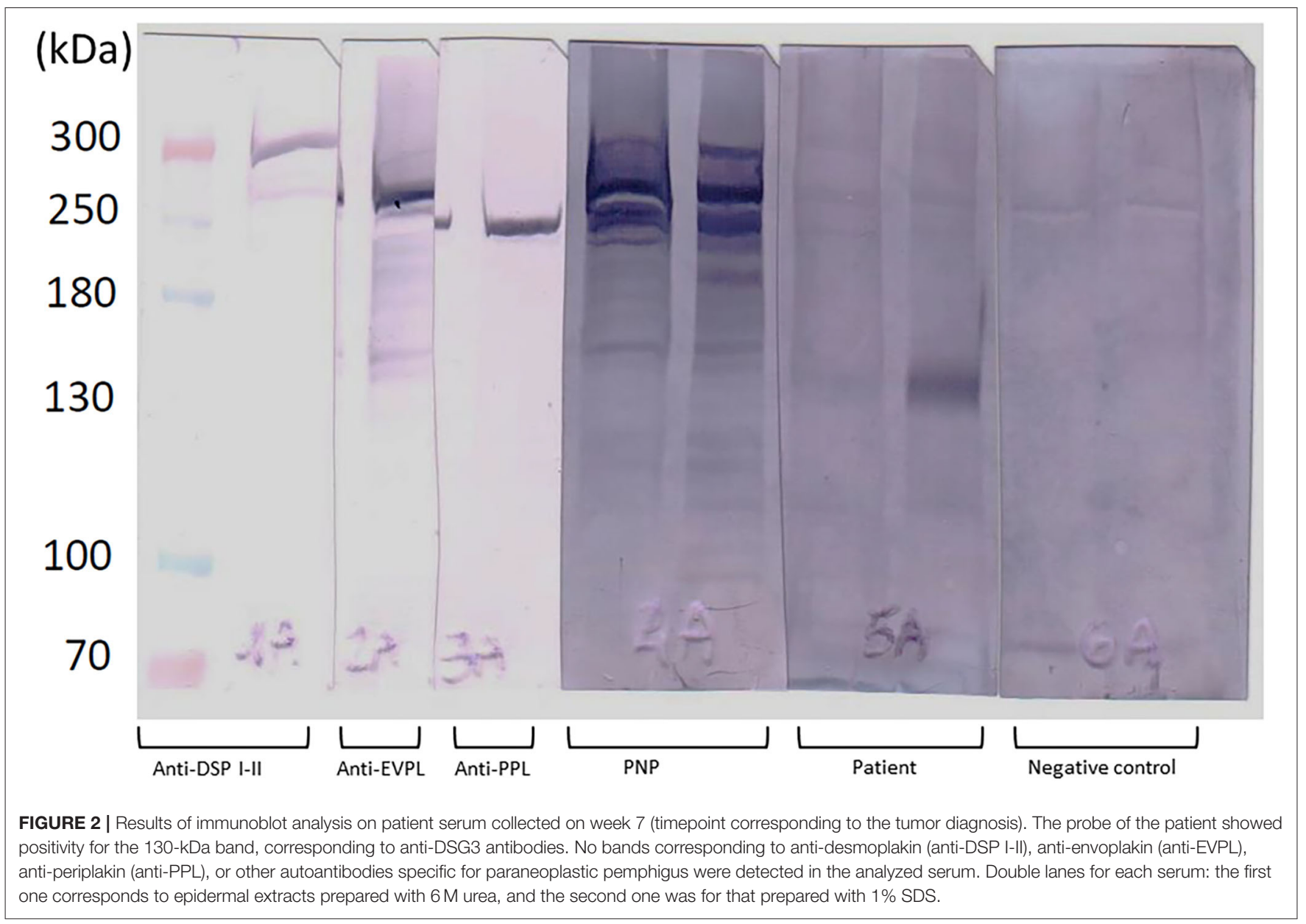

The patient underwent robot-assisted Ivor-Lewis esophagectomy with latero-lateral anastomosis and jejunostomy feeding tube (J-tube) placement. Tissue perfusion and esophagogastric anastomosis evaluation with the indocyanine green method were performed. After the surgery, the patient achieved early mobilization, with no signs of anastomotic leak at endoscopies and CT scan. The post-operative course was uneventful, except for an episode of atrial fibrillation, which was managed with two subsequent doses of verapamil according to the advice of the cardiologist. The patient was then discharged on post-operative day 11, with no dysphagia nor nausea reported. The histopathologic examination of the esophageal mass confirmed the diagnosis of gastric cardia adenocarcinoma.

Despite the successful surgical management of the tumor, PV continued to worsen, with blisters and erosions affecting the entire body, except for mucosal sites and feet (Figure 3).

Considering the recommendation to avoid high-dose systemic steroids also after surgery, due to the risk of anastomotic leakage, the patient was managed with 4 weekly infusions of rituximab at a dose of $500 \mathrm{mg}$ (lymphoma protocol). A moderate-dose systemic steroid therapy (prednisone 0.6 $\mathrm{mg} / \mathrm{kg} /$ day) was started only 2 weeks later in concomitance with the end of the national lockdown.
The skin of the patient started improving progressively, with complete resolution of the lesions. The anti-DSG1 and anti-DSG3 titers likewise decreased progressively (Figure 4).

At the 4-month dermatologic follow-up, only postinflammatory hyperpigmentation or hypopigmentation was detectable, with no active lesions (Figure 3), while the last enzyme-linked immunosorbent assay (ELISA) performed resulted in lower anti-desmoglein autoantibody concentrations. No surgery-related criticalities or tumor recurrences were highlighted during this period.

\section{METHODS}

\section{Anti-Desmoglein Enzyme-Linked Immunosorbent Assay}

Assessments of circulating anti-DSG1 and anti-DSG3 autoantibodies were performed through ELISA using commercial kits (MBL MESACUP-2 TEST, Naka-Ku Nagoya Aichi, Japan). The kit was composed of microwells which were coated with recombinant DSG1 and DSG3. The cutoff values for positivity were $14 \mathrm{UI} / \mathrm{ml}$ for anti-DSG1 and $7 \mathrm{UI} / \mathrm{ml}$ for anti-DSG3, according to the indication of the manufacturer. 


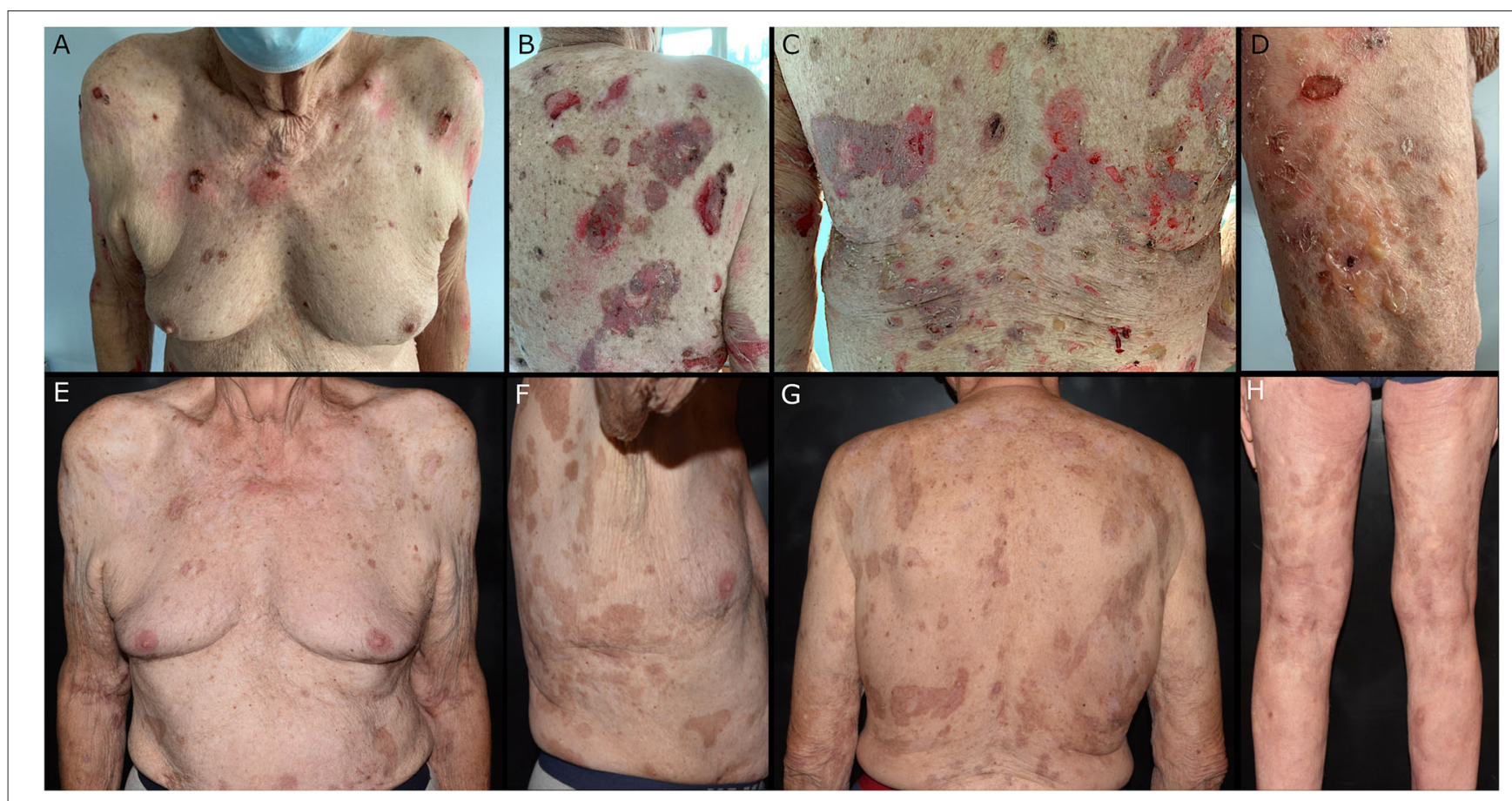

FIGURE 3 | (A-D) Soft blisters and painful erosion affecting the trunk and limbs a few days after the surgical removal of the esophageal mass. (E-H) Residual post-inflammatory hyperpigmentation in sites previously affected by pemphigus lesions at the 4-month follow-up.

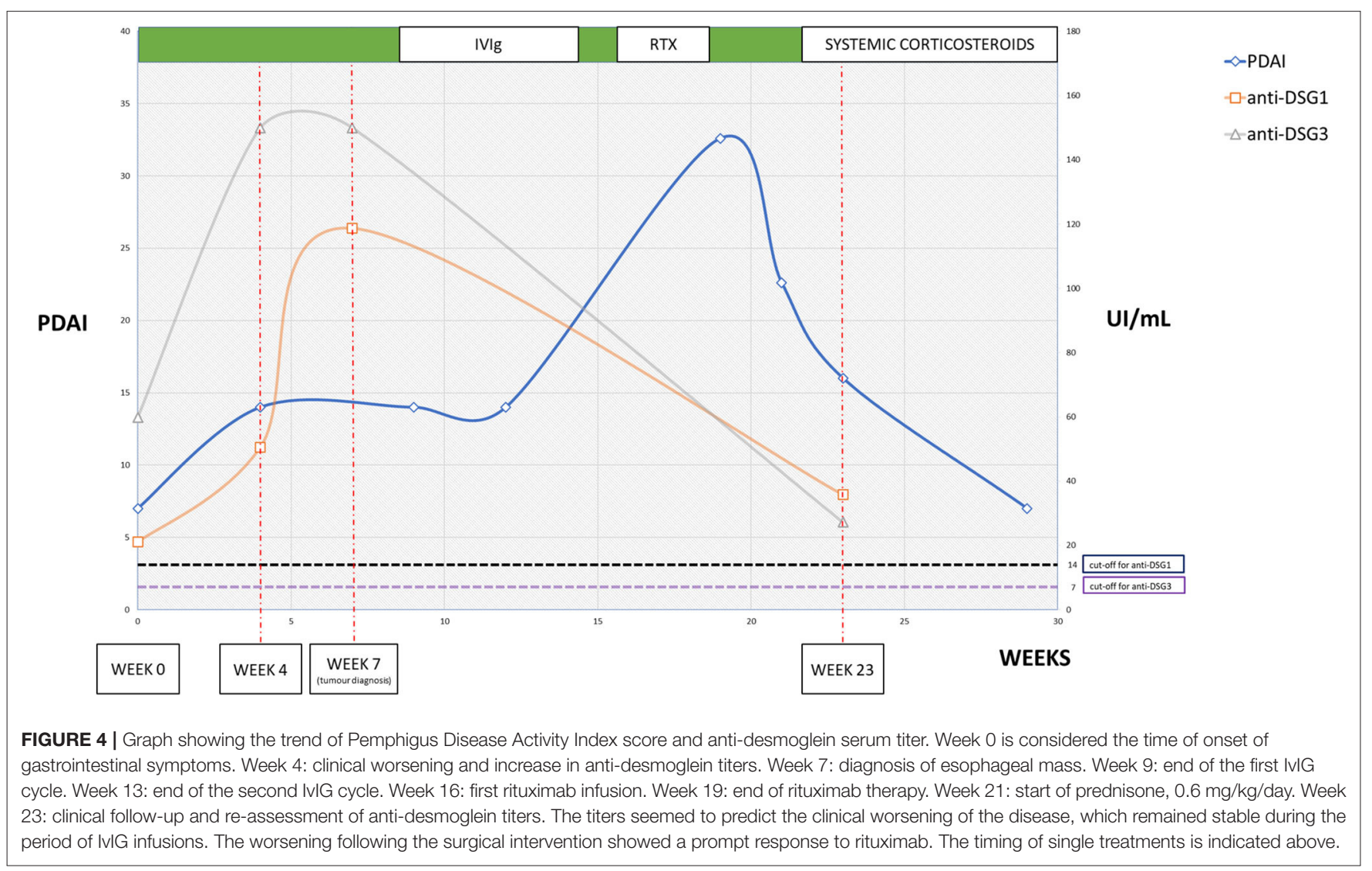




\section{Envoplakin ELISA}

Assessment of circulating anti-envoplakin autoantibodies was performed through ELISA using commercial kits (Euroimmun, Lubeck, Germany), according to the protocol of the manufacturer. The cutoff values for envoplakin positivity was 18.6 UI.

\section{Indirect Immunofluorescence}

IIF on rat bladder was performed using a commercial kit (FA 1507-1005, EUROIMMUN AG, Lübeck, Germany) containing rat bladder slides and fluorescein isothiocyanate-conjugated antihuman IgG secondary antibodies and then examined under a Nikon C2 confocal microscope. Indirect Immunofluorescences were also performed on normal human skin substrate with patient sera collected at timepoints week $0,4,7$, and 23 .

\section{Immunoblot}

SDS-polyacrylamide gel electrophoresis was performed using $6 \%$ polyacrylamide gel loaded with epidermal extracts prepared with $6 \mathrm{M}$ urea (left lane) and 1\% SDS (right lane) for patient and control sera, and epidermal extracts were prepared with urea for control antibodies in reducing conditions. After transfer to polyvinylidene difluoride membrane (Immobilon-P, Millipore, USA) and blocking in 5\% non-fat milk (Merck, Germany), immunoreactivity was detected by incubation with 1:20 and 1:100 dilution of patient and control sera and antibodies to Desmoglein 1 (Santa Cruz, California), Desmoglein 3 (R\&D, Minneapolis), Desmocollin 1 (Progen, Germany), Desmocollin 2 (Progen, Germany), Desmoplakin I and II (Progen, Germany), Envoplakin (Santa Cruz, California), and Periplakin (Santa Cruz, California). After washing, the membrane was incubated with alkaline phosphatase-labeled secondary antibody against IgG (Southern Biotech, USA and CA). Then, it was washed again and stained with chromogenic substrates, 5-bromo-4-chloro-3indolyl-phosphate and nitroblue tetrazolium (Roche, Swiss).

\section{RESULTS}

Different ELISAs for detecting anti-DSG1 and anti-DSG3 autoantibodies were performed (Figure 4). At week 0, the antiDSG1 titer was $21.1 \mathrm{UI} / \mathrm{ml}$ and the anti-DSG3 titer was 59.9 $\mathrm{UI} / \mathrm{ml}$. At week 4 , the values were significantly increased as follows: $50.6 \mathrm{UI} / \mathrm{ml}$ for anti-DSG1 and $>150 \mathrm{UI} / \mathrm{ml}$ for antiDSG3. At week 7, the anti-DSG1 was $118.7 \mathrm{UI} / \mathrm{ml}$, while the anti-DSG3 was $>150 \mathrm{UI} / \mathrm{ml}$. The values then decreased to $35.8 \mathrm{UI} / \mathrm{ml}$ for anti-DSG1 and $27.2 \mathrm{UI} / \mathrm{ml}$ for anti-DSG3 by week 23. The ELISA for envoplakin was performed on the same sera collected and showed negative results, as shown in Supplementary Figure 1B.

No staining was visible with a confocal microscope on rat bladder indirect immunofluorescence performed on weeks 0,4 , 7, and 23 sera (Supplementary Figure 3).

Patient sera showed positivity for autoantibodies targeting the $130-\mathrm{kDa}$ band on immunoblot analysis, which corresponds to DSG3. No reactivity at bands corresponding to plakin family or desmocollin family proteins was noted (Figure 4;
Supplementary Figure 1A). The investigations were performed on serum collected at weeks $0,4,7$, and 23 .

\section{DISCUSSION}

This is a case of PV worsened in concomitance with the development of a solid gastrointestinal tumor. Pemphigus diagnosis was made several years before the detection of the neoplasm, which therefore may represent an exacerbating factor for the bullous disease rather than of inductor. Relapses of PV after rituximab treatment are not infrequent, involving up to $63 \%$ of patients even years after a single cycle of therapy, while the rate seems to be low in patients also treated with adjunctive maintenance rituximab administrations (2). However, the timing raised the suspicion of PNP (3), an autoimmune mucocutaneous disease for which Nguyen et al. in 2001 proposed the term "paraneoplastic autoimmune multiorgan syndrome" (PAMS), in consideration of the frequent multi-systemic involvement (4). Although the correlation with malignancy seemed to be clear, PNP/PAMS diagnosis was excluded for different reasons. Mucosal and respiratory tract involvement was completely absent, while skin lesions resembled a classical PV blister and erosions. Moreover, IIF on rat bladder revealed repeatedly negative results, as well as immunoblot for antienvoplakin and anti-desmocollin autoantibodies. Serological analysis was performed on sera collected at different timepoints (Supplementary Figure 1): week 0 is considered the time of onset of tumor-related symptoms, week 4 consists in the subsequent patient visit, week 7 is the time of the tumor diagnosis, and week 23 represents the first follow-up after the rituximab cycle (Figure 4). The correlation between PV and malignancies is well-established, with malignancies reported in showing a role in induction or exacerbation of a previously diagnosed PV $(5,6)$. Hematologic malignancies seem to have a higher incidence among PV patients $(7,8)$, but a large-population study also evidenced the association with oropharyngeal and colon carcinomas, which were present in 0.9 and $3.7 \%$ of PV patients, respectively. In general, gastrointestinal cancers were present in $6.1 \%$ of patients, compared to the $2.8 \%$ of the control population. In addition, a large populationbased study found a significant association between PV and esophageal cancer (9). The patient described in this case had a history and clinical presentation consistent with PV, considering the lack of severe mucosal involvement and serological investigations. Despite the serological positivity for both anti-Dsg1 and anti-Dsg3 IgG, the patient never showed mucosal involvement, while the cutaneous lesions were clinically more compatible with PV than with pemphigus foliaceous. Interestingly, other authors hypothesized the existence of a "cutaneous-type" PV, describing similar cases where the absence of mucosal lesions could be due to the low pathogenetic activity of anti-Dsg3 not sufficient to induce blistering as in the mucocutaneous or mucosal-dominant phenotypes of PV $(10,11)$.

The management of the patient required an urgent surgical excision of the neoplasm due to the symptoms triggered 
and the potentially life-threatening course of the disease. Pemphigus-specific immunosuppressive treatments had to be post-poned in order to avoid perioperative risks and infective complications. In fact, a prolonged high-dosed systemic steroid therapy could have a detrimental effect on the healing process of anastomosis, raising the leakage risk $(12,13)$. Moreover, the concomitant peak of COVID-19 pandemic in Italy emphasized the need to reduce the infective risk. Thus, surgeons and dermatologists temporarily post-poned the anti-CD20 therapy to reduce the level of immunosuppression. The choice of administering IVIg was guided by the evidence of effectiveness in both pemphigus and COVID-19 disease $(14,15)$.

This case highlights the possibility of hidden neoplasms in patients experiencing a severe and sudden worsening of pemphigus. The physiopathology of this phenomenon is still unknown and is probably a complex relationship which needs further studies. The association between cancer and autoimmunity is bidirectional, as different autoimmune disorders can promote tumorigenesis; malignancies may likewise increase the risk of autoimmune diseases (16). Tissues affected by an autoimmune disease may present localized reduction of self-tolerance, leading to chronic inflammation and oncogenesis (17). On the other hand, neoantigens expressed early in cancer may elicit an immune response, enhancing autoimmune disease activity indirectly through $\mathrm{B}$ cell maturation and proliferation with increased autoantibody production.

It is clear that the generation of many abnormal antigens during tumorigenesis can be recognized early by the immune system, thus triggering a reaction known as cancer immunoediting, which can induce the host immune response to produce autoantibodies in the early phase before the clinical manifestations. This induced researchers to investigate these autoantibodies as early diagnostic biomarkers for esophageal squamous cell carcinoma and esophagogastric junction adenocarcinoma (18).

Considering the possible association between pemphigus and cancer may lead to an earlier tumor diagnosis, thus influencing the survival rate and treatment regimen. The management of severe PV in oncologic patients poses significant challenges due to the risk of immunosuppressive treatments and absence of dedicated guidelines. Our patient required rituximab therapy due to the lack of sufficient disease control by IVIg treatment and the need to avoid a high dose of systemic corticosteroids. The high efficacy of rituximab in the short term counterbalanced the risk of tumor progression with prolonged B-cell depletion. A close collaboration between dermatologists, surgeons, and oncologists is needed to adopt the best treatment strategy in order to improve patient outcome and quality of life.

\section{PERSPECTIVE OF THE PATIENT}

The patient was obviously scared about the diagnosis of esophageal adenocarcinoma and afraid of the possibility of dying soon. Subsequently, the worsening of pemphigus caused the well-known sensations of burning and extreme pain in a large part of the total skin surface, impairing his quality of life and the normal activities of daily living. During the treatment with IVIg, the distress was so intense that the patient said repeatedly that it was the most painful sensation of his life. Besides that, the risk of tumor progression and the known low survival rate of advanced esophageal cancer convinced him to undergo the surgical intervention, along with the awareness of the slow progression of symptoms which could lead to the incapability of feeding.

The successful surgical intervention represented a strong signal for him, particularly due to the early discharge with prompt recovery of normal alimentation. The positivity derived from this part of the therapeutic course allowed him to face the treatment with rituximab with more determination, focusing on the possibility to get a full recovery.

\section{DATA AVAILABILITY STATEMENT}

The original contributions generated for the study are included in the article/Supplementary Material, further inquiries can be directed to the corresponding author.

\section{ETHICS STATEMENT}

Written, informed consent was obtained from the patient for the publication of this case report.

\section{AUTHOR CONTRIBUTIONS}

$\mathrm{MC}, \mathrm{AC}, \mathrm{LQ}, \mathrm{AV}, \mathrm{CA}$, and $\mathrm{MM}$ were directly involved in patient management, produced and drafted the manuscript and revisioned the literature. MC concepted the whole work and revised carefully the manuscript. OL collected radiological images and revised the manuscript. FCa, GB, FCo, and FCi were involved in the surgical aspect of patient management, revised the manuscript, and collected histopathological pictures. AS, GD, and BB provided immunoserological analysis, collected the images of immunoblot, and indirect immunofluorescence. All authors contributed to the article and approved the submitted version.

\section{SUPPLEMENTARY MATERIAL}

The Supplementary Material for this article can be found online at: https://www.frontiersin.org/articles/10.3389/fmed. 2021.708284/full\#supplementary-material

Supplementary Figure 1 | Serological tests for patient diagnosis. (A) Immunoblotting on epidermal extracts did not show any significant (absent in controls) bands for PNP diagnosis. Lane 1, anti-desmoglein 1 (160 kDa); lane 2, anti-desmoglein 3 (130 kDa); lane 3, anti-desmocollin 1 (110 kDa); lane 4, anti-desmocollin 2 (120 kDa); lane 5, anti-envoplakin (210 kDa); lane 6, anti-periplakin (190 kDa); lane 7, anti-desmoplakin 1/2 (250 and $210 \mathrm{kDa}$ ); lane 8, paraneoplastic pemphigus serum (positive control); lanes 9-12, sera of the patient at weeks 0, 4, 7, and 23, respectively; lanes 13-15, negative control sera. (B) Sera from the present case did not react with envoplakin by ELISA. Paraneoplastic pemphigus serum (positive control) and sera of the patient at weeks $0,4,7$, and 23 were tested by envoplakin ELISA. Cutoff value $=18.6$ units (dashed line).

Supplementary Figure 2 | Indirect immunofluorescence on normal human skin performed on patient serum collected at weeks $0,4,7$, and 23 . The sera were tested for both $\lg G$ and $\lg A$ at each timepoint with 1:20 dilution for $\lg G$ and 1:10 
for $\lg A$. The pictures show mild-moderate positive staining for $\lg G$, while $\lg A$ was negative in all timepoints.

Supplementary Figure 3 | Indirect immunofluorescence performed on rat bladder substrate at the same timepoints (weeks 0, 4, 7, and 23). All the probes resulted negative compared to the positive control.

\section{REFERENCES}

1. Bray F, Ferlay J, Soerjomataram I, Siegel RL, Torre LA, Jemal A. Global cancer statistics 2018: GLOBOCAN estimates of incidence and mortality worldwide for 36 cancers in 185 countries. CA Cancer J Clin. (2018) 68:394-424. doi: $10.3322 /$ caac. 21492

2. Shimanovich I, Baumann T, Schmidt E, Zillikens D, Hammers CM. Long-term outcomes of rituximab therapy in pemphigus. J Eur Acad Dermatol Venereol. (2020) 34:2884-9. doi: 10.1111/jdv.16561

3. Kim JH, Kim SC. Paraneoplastic pemphigus: paraneoplastic autoimmune disease of the skin and mucosa. Front Immunol. (2019) 10:1259. doi: 10.3389/fimmu.2019.01259

4. Nguyen VT, Ndoye A, Bassler KD, Shultz LD, Shields MC, Ruben BS, et al. Classification, clinical manifestations, and immunopathological mechanisms of the epithelial variant of paraneoplastic autoimmune multiorgan syndrome: a reappraisal of paraneoplastic pemphigus. Arch Dermatol. (2001) 137:193-206.

5. Streifel AM, Wessman LL, Schultz BJ, Miller D, Pearson DR. Refractory mucositis associated with underlying follicular dendritic cell sarcoma of the thymus: paraneoplastic pemphigus versus malignancyexacerbated pemphigus vulgaris. JAAD Case Rep. (2019) 5:933-6. doi: 10.1016/j.jdcr.2019.09.009

6. Sendrasoa FA, Ranaivo IM, Rakotoarisaona MF, Raharolahy O, Razanakoto $\mathrm{NH}$, Andrianarison $\mathrm{M}$, et al. Pemphigus vulgaris as the first manifestation of multiple myeloma: a case report. J Med Case Rep. (2018) 12:255. doi: 10.1186/s13256-018-1791-Z

7. Kridin K, Zelber-Sagi S, Comaneshter D, Batat E, Cohen AD. Pemphigus and hematologic malignancies: a population-based study of 11,859 patients. J Am Acad Dermatol. (2018) 78:1084-9.e1. doi: 10.1016/j.jaad.2017. 11.039

8. Schulze F, Neumann K, Recke A, Zillikens D, Linder R, Schmidt E. Malignancies in pemphigus and pemphigoid diseases. I Invest Dermatol. (2015) 135:1445-7. doi: 10.1038/jid.2014.547

9. Kridin K, Zelber-Sagi S, Comaneshter D, Cohen AD. Coexistent solid malignancies in pemphigus: a population-based study. JAMA Dermatol. (2018) 154:435-40. doi: 10.1001/jamadermatol.2017. 6334

10. Yoshida K, Takae Y, Saito H, Oka H, Tanikawa A, Amagai M, Nishikawa T. Cutaneous type pemphigus vulgaris: a rare clinical phenotype of pemphigus. $J$ Am Acad Dermatol. (2005) 52:839-45. doi: 10.1016/j.jaad.2005.01.106

11. Gheisari M, Shahidi-Dadras M, Nasiri S, Dargah SM, Dadkhahfar S, Abdollahimajd F. Cutaneous type of pemphigus vulgaris. J Am Acad Dermatol. (2020) 83:919-20. doi: 10.1016/j.jaad.2019.12.065
Supplementary Figure 4 | Histological pictures of adenocarcinoma of the gastro-esophageal junction. (A) Tubular and solid pattern gastric adenocarcinoma infiltrating submucosal tissue (magnification $\times 100$, hematoxylin and eosin). (B) Lauren intestinal-type gastric adenocarcinoma; infiltrative glands with different (moderate and low) grades of differentiation (original magnification $\times 200$, hematoxylin and eosin).

12. Van Daele E, Van de Putte D, Ceelen W, Van Nieuwenhove Y, Pattyn P. Risk factors and consequences of anastomotic leakage after Ivor Lewis oesophagectomy. Inter Cardiovasc Thorac Surg. (2016) 22:32-7. doi: 10.1093/icvts/ivv276

13. Kassis ES, Kosinski AS, Ross P, Jr., Koppes KE, Donahue JM, Daniel VC. Predictors of anastomotic leak after esophagectomy: an analysis of the society of thoracic surgeons general thoracic database. Ann Thorac Surg. (2013) 96:1919-26. doi: 10.1016/j.athoracsur.2013.07.119

14. Amagai M, Ikeda S, Shimizu H, Iizuka H, Hanada K, Aiba S, et al. A randomized double-blind trial of intravenous immunoglobulin for pemphigus. J Am Acad Dermatol. (2009) 60:595-603. doi: 10.1016/j.jaad.2008.09.052

15. Galeotti C, Kaveri SV, Bayry J. Intravenous immunoglobulin immunotherapy for coronavirus disease-19 (COVID-19). Clin Transl Immunology. (2020) 9:e1198. doi: 10.1002/cti2.1198

16. Giat E, Ehrenfeld M, Shoenfeld Y. Cancer and autoimmune diseases Autoimmun Rev. (2017) 16:1049-57. doi: 10.1016/j.autrev.2017.07.022

17. Castro FA, Liu X, Försti A, Ji J, Sundquist J, Sundquist K, et al. Increased risk of hepatobiliary cancers after hospitalization for autoimmune disease. Clin Gastroenterol Hepatol. (2014) 12:1038-45.e7. doi: 10.1016/j.cgh.2013.11.007

18. Xu YW, Peng YH, Xu LY, Xie JJ, Li EM. Autoantibodies: potential clinical applications in early detection of esophageal squamous cell carcinoma and esophagogastric junction adenocarcinoma. World J Gastroenterol. (2019) 25:5049-68. doi: 10.3748/wjg.v25.i34.5049

Conflict of Interest: The authors declare that the research was conducted in the absence of any commercial or financial relationships that could be construed as a potential conflict of interest.

Publisher's Note: All claims expressed in this article are solely those of the authors and do not necessarily represent those of their affiliated organizations, or those of the publisher, the editors and the reviewers. Any product that may be evaluated in this article, or claim that may be made by its manufacturer, is not guaranteed or endorsed by the publisher.

Copyright (c) 2021 Corrà, Cammelli, Quintarelli, Barbato, Le Rose, Salemme, Di Zenzo, Coratti, Verdelli, Aimo, Mariotti, Bianchi, Cianchi and Caproni. This is an open-access article distributed under the terms of the Creative Commons Attribution License (CC BY). The use, distribution or reproduction in other forums is permitted, provided the original author(s) and the copyright owner(s) are credited and that the original publication in this journal is cited, in accordance with accepted academic practice. No use, distribution or reproduction is permitted which does not comply with these terms. 